\title{
A Study on Threats Detection and Tracking Systems for Military Applications using WSNs
}

\author{
Tareq Alhmiedat \\ Dept. of Computer Science, \\ Zarqa University, \\ P.O. Box 132222, \\ Zarqa 13132, Jordan
}

\author{
Anas Abu Taleb \\ Dept. of Computer Science, \\ Isra University, \\ P.O. Box 22, 33, \\ Amman 11622, Jordan
}

\author{
Mohammad Bsoul \\ Dept. of Computer Science, \\ The Hashemite University, \\ P.O. Box 150459, \\ Zarqa 13115, Jordan
}

\begin{abstract}
In Wireless Sensor Network (WSN) applications it is critical to accurately determine the location of the distributed sensor nodes in order to report the data that is geographically meaningful. Since localization and tracking algorithms have been attracting research and development attention recently, a wide range of existing approaches regarding this topic have emerged. Tracking and localization algorithms have been proposed for different WSN applications including civilian, industrial and safety applications. A few research studies focused on tracking Threats through military applications, such as detecting and tracking Threats through border security area. Therefore this paper studies and investigates the existing WSN based tracking and localization algorithms and summarizes the potential requirements for localizing and tracking Threats through military applications. The existing systems are categorized and discussed. A critical analysis is found in this paper, in order to guide the developer to design and implement a WSN-based tracking system for military applications.
\end{abstract}

\section{General Terms}

Wireless Sensor Networks.

\section{Keywords}

Tracking, Localization, Acoustic, Military applications.

\section{INTRODUCTION}

Wireless Sensor Network (WSN) has become a vital research area, due to their wide ranging applications. WSN based systems have been deployed widely in many applications including civilian, industrial, agricultural, and military applications [1-5].

A sensor network is composed of sensor nodes which are small in size, low in cost, and have short communication range. A sensor node usually consists of four sub-systems:

a. A computing subsystem: this is responsible for functions such as execution of the communication protocols and control of sensors,

b. A sensing subsystem: this subsystem is responsible for sensing the environmental characteristics, such as using temperature, humidity, or acoustic sensor,

c. A communication subsystem: this consists of a short radio range used to communicate with neighboring nodes, d. A power supply subsystem: this includes a battery which provides energy to sensor node.

Researchers have focused on different aspects of WSN, such as hardware design, routing, data aggregation, and localization. One of the critical issues which needs to be taken into consideration is localizing objects through distributed sensor network. Node localization is the problem of finding the geographical location of each target node (the object with unknown location) based on other reference nodes (nodes with known location). Localizing sensor nodes is one of the fundamental and difficult problems that must be solved for WSN. Tracking and localization systems have been deployed to track civilian, soldiers, and animals. Figure 1 depicts the idea of tracking mobile targets through WSNs, and transmit the localization information to a base station.

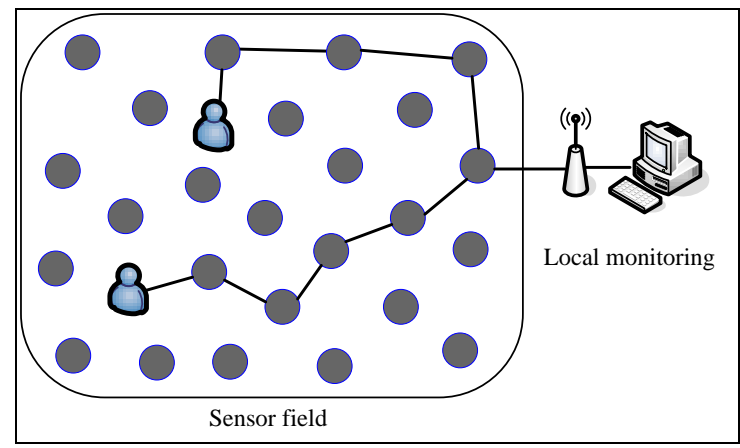

Fig 1: The idea of tracking mobile targets through WSNs

The WSN based localization and tracking issues have received much attention recently driven by the need to achieve high localization accuracy with the minimum cost. This is because:

a. In several applications, the location itself is the information of interest,

b. Transferring sensors' measurements without incurring the sensors' locations is an ineffective task,

c. Several routing protocols are based on the locations of sensor nodes.

There are many issues affecting the efficiency of the tracking system including: the cost of extra localization hardware, number of reference nodes in the tracking area, and the communication range for the target and reference nodes. These issues have to be taken into account before developing a WSN-based tracking system. 


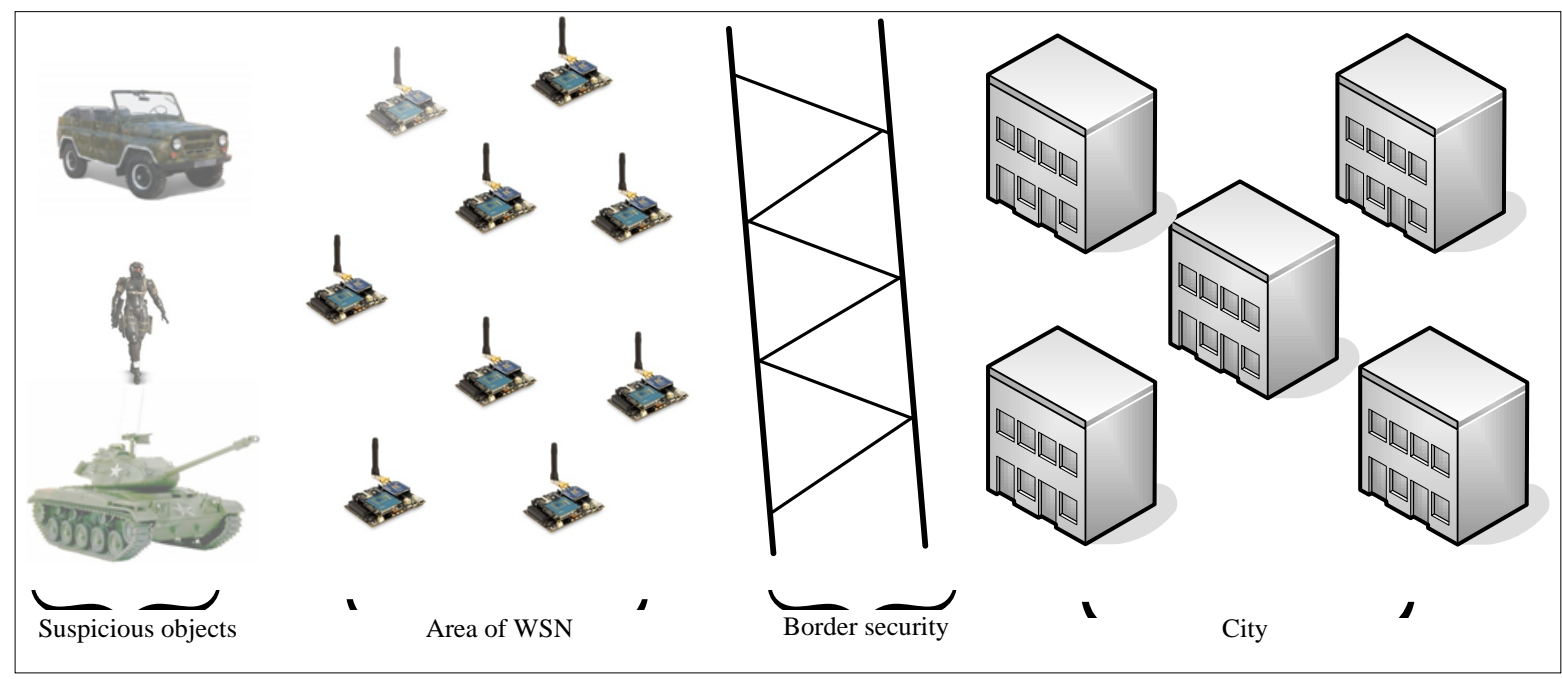

Fig 2: The idea of Threats detection and tracking using WSN

A wide range of node localization and tracking systems have been proposed recently. According to [6], most of the WSNbased localization solutions are either range-based [7-10] or range-free [11-13]. Range-based approaches deploy various techniques (ultrasound, infrared, and GPS devices) to first determine the distance between reference and target nodes, and then compute the location using geometric principles. In order to calculate the distance between the target and reference nodes, an additional hardware is usually required to be attached to each wireless sensor device.

On the other hand, in range-free approaches, distances are not computed directly, as the number of hops between the target and reference nodes is calculated. As soon as the hop counts are calculated, distances between nodes (reference and target nodes) are computed based on the average distance per hop. And then, geometric principles are used to calculate the target's location. Range-free techniques are not accurate as range-based ones and often require a high number of messages to be transmitted before calculating the target's locations [14]. Since, range-based systems require attaching additional hardware to each target and reference nodes, which increases both the cost and power consumption for sensor nodes. But, range-based approaches offer better localization accuracy than the range-free systems. Furthermore, range free approaches require scattering a large number of reference nodes in order to offer efficient localization accuracy [15]. Therefore, this paper focuses on range based systems as they offer better localization accuracy than range free systems.

The existing WSN-based localization systems are discussed in details in [16]. However, in this paper, we aim to discuss the existing WSN based localization approaches which could be used for Threats detection and tracking in military applications, and point out the key issues which need to be taken into consideration before designing and implementing a WSN based localization approach for Threats detection and tracking. The idea of the research's goal is depicted in Figure 2. Our contribution lies on the following aspects:

i. Study the existing WSN-based localization and tracking systems,

ii. Present, discuss, and compare WSN based localization and tracking methods which could be deployed in military applications, iii. Present the key issues which need to be taken into consideration before designing and developing a WSNbased localization system for Threats detection and tracking applications.

This paper is organized as follows: Section 2 presents a classification of WSN-based tracking and localization. Section 3 discusses the strengths and weaknesses of the existing localization and tracking systems. Recommendations for WSN developers in order to design and implement a WSN-based tracking system for military applications are shown in Section 4. And finally, conclusions and future work presented in Section 5.

\section{CLASSIFICATION OF WSN-BASED LOCALIZATION SYSTEMS}

Today, there are a wide variety of wireless sensor devices available in market that are introduced and compared in [17]. In WSN, localization and tracking algorithms usually focus on the aspect of sensor nodes' interaction with the target after the target has been detected within the area of interest. Once the target node is detected, the reference nodes collect localization information and then use one of many different types of algorithms to calculate the target's location. From here, it is the goal of the reference nodes in the sensor network to track the target node as it moves through the network. Several of these tracking systems are discussed in details in [16]. Now, we focus on tracking and localization technologies which can be used to detect and track mobile Threats through WSNs. In this paper, we categorize the WSN-based military tracking systems into four main categories as shown in Figure 3.

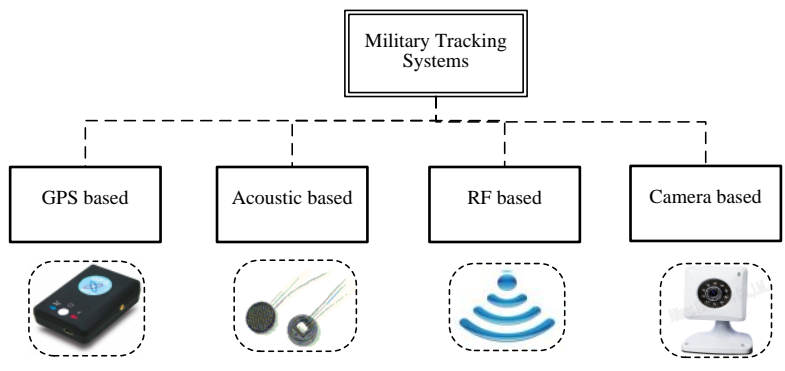

Fig 3: Classification of WSN based military tracking systems 


\subsection{GPS-based Systems}

The Global Positioning System (GPS) was originally designed for military applications. Figure 4 presents a GPS receiver device. However, it is used now by civilian users since 1983 . The GPS satellites transmit signals to the GPS receiver. The GPS receiver obtains the signals transmitted by the GPS satellites, and computes its current location. GPS operations depend on a very precise time reference, which is provided by atomic clocks on board of satellites. GPS receivers do not transmit and require a line of sight between the GPS satellites and receivers, and therefore, GPS-systems work effectively outdoors.

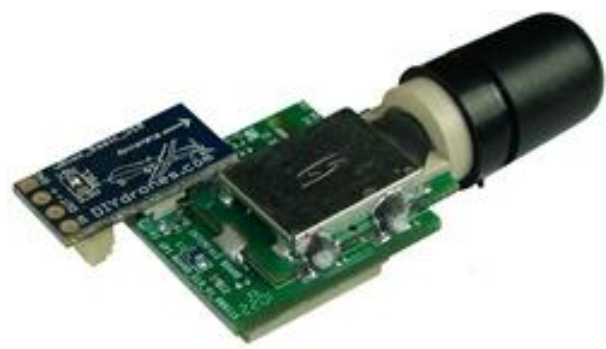

Fig 4: GPS receiver

GPS-based tracking systems require attaching a GPS receiver and transceiver to each target object. The GPS receiver obtains the location information from satellites, while the transceiver is used to transmit the localization information to a base station. As depicted in Figure 5, the vehicle obtains the location information from the satellites, then computes its current location, and then transmits the localization information to a base station using a Global System of Mobile Communications (GSM) network.

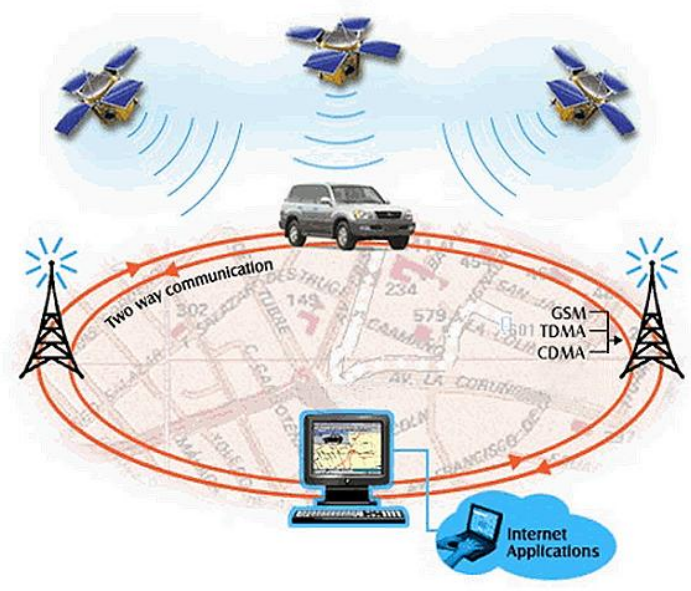

Fig 5: A GPS-based tracking system

Recently, GPS-based tracking systems have been integrated with WSN in order to enhance the localization accuracy for tracking mobile targets moving through the wireless sensor's area of interest as proposed in [18, 19]. Furthermore, GPS systems have been deployed in environmental and monitoring applications, as in the system proposed in [20] which combines GPS collars and satellite images with WSN to monitor behavioral preferences and social behaviors of cattle using a GPS technology. Moreover, a GPS-based system is proposed in [21] to monitor nuclear radiation through WSN.

\subsection{Visual-based Systems}

Visual-based localization systems are a natural sensing modality for tracking applications, since each target does not require carrying or wearing any special device. These systems track mobile targets based on their presence or absence. Camera-based systems have been used for tracking and monitoring objects through WSN [17, 22-25]. Figure 6 presents a sensor node with an attached camera.

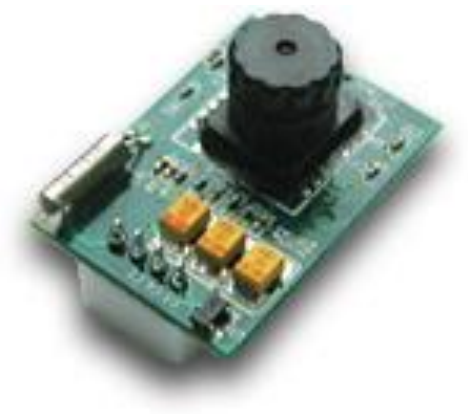

Fig 6: A camera-based wireless sensor device

\subsection{Radio Frequency (RF) based Systems}

Radio Frequency (RF) tracking systems are based on the use of radio waves to determine the target's location. Radio signal information from a wireless transmitter can be used to estimate the location of target nodes in two ways: the first uses the signal propagation model to convert signal strength (SS) to a distance measurement, using previous knowledge about reference nodes' coordinates, and deploys a geometry method to compute the location for target nodes. This is known as a triangulation localization method [11, 26-28]. The second method uses the behavior of signal propagation and information about the geometry of a building to convert RSS values into distance values; this is known as a fingerprinting localization method [7, 29-33].

\subsection{Acoustic-based Systems}

Acoustic-based localization systems include computing the distance between a reference node and a target node based on acoustic behaviors. These techniques have received much attention recently, as they offer precise localization information. However, this kind of tracking systems necessitates a real voice revealed by the target object, in order to be able to be tracked. Acoustic-based systems have been deployed in WSN based tracking applications; Figure 7 presents an acoustic sensor node (a microphone attached to a wireless sensor node).

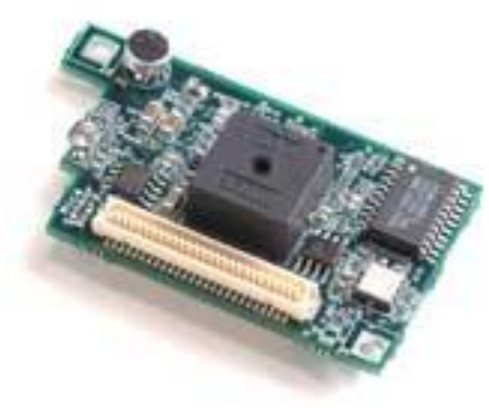

Fig 7: Acoustic wireless sensor device

In acoustic WSN based tracking systems [5, 34-38], reference nodes themselves do not need to generate an acoustic signal for ranging, and only the mobile target needs to emit acoustic signals. Figure 8 depicts a WSN based tracking system to detect and track the positions of a special kind of birds [39]. 


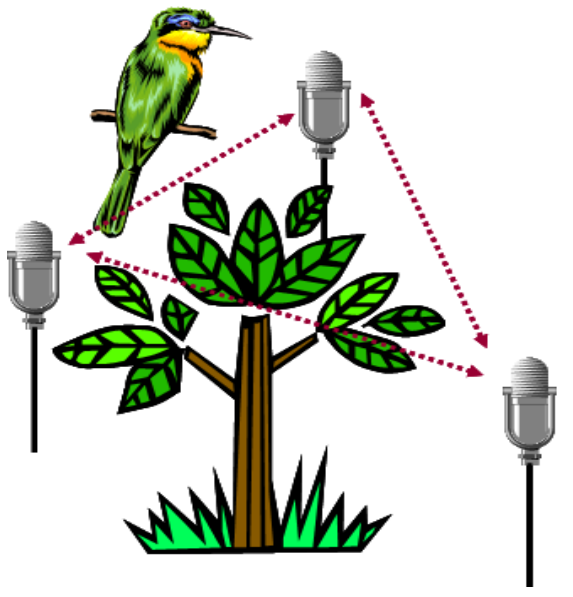

Fig 8: Tracking birds using acoustic devices

\section{DISCUSSION AND CRITICAL ANALYSIS}

WSN-based tracking system can be used to offer critical tasks for military applications, such as localizing and tracking mobile targets (vehicles, and soldiers). Practically, designing and developing a localization method for WSN is a complicated task due to the limited capabilities of sensor devices. The analysis of the existing WSN localization and tracking systems is presented in this section.

\subsection{Analysis of the Existing WSN based Approaches}

In this section, we study and compare the efficiency of the existing systems in terms of their strengths and weaknesses. We start with GPS-based approaches. GPS-based systems offer reasonable localization accuracy and have been deployed in several applications including military, civilian and industrial. However, using GPS-based systems for Threats detection and tracking tasks is an inefficient for several reasons: First, each threat object is required to be attached with a GPS receiver and transceiver, and this is not applicable in military Threats detection and tracking applications.

Second, GPS systems require a straight line of sight between the receiver and GPS satellites, and this requirement is not valid for indoor tracking, due to the obstacles and walls found between the senders and receivers. And third, attaching additional hardware to each sensor node will increase both the sensor's size and cost. Therefore, GPS-based systems are considered as inefficient tracking system for Threats detection and tracking applications.

Let's move to the second approach discussed in the literature, the camera-based approach. Camera-based systems offer efficient localization accuracy, as these systems track positions and identities of mobile targets, without the need for attaching any device to suspect objects. The same with GPS approaches, camera-based systems suffer from a high cost, in addition to the requirement of a straight line of sight to be existed between the camera (reference node) and target object, in order to compute the targets' locations. One the other hand, camera-based approaches require to be used in day time in order to be able to detect the targets' positions. A night camera can be used to track objects; however this solution is an ineffective in terms of cost. Consequently, using the camera approach in WSN-based tracking system is not a practical tracking solution for several reasons: a. The requirement of installation and maintenance, including periodic lens cleaning,

b. Performance affected by inclement weather such as fog, rain, and snow,

c. Reliable night-time signal actuation requires street lighting,

d. Some models are susceptible to camera motion caused by strong winds or vibration of camera mounting structure.

RF-based approach was the third approach taken into consideration for detecting and tracking Threats. RF-based systems can be deployed to track targets with low cost, as there is no need to attach special hardware to each reference and target wireless sensor nodes. These approaches offer costwise localization solutions, but offering low localization accuracy. RF-based systems don't require attaching any additional device or sensor to each target node except the transmitter and receiver found at each sensor device; however the sensor's cost is not expensive as in the camera and GPSbased systems.

The last approach discussed in the literature was the acousticbased approach. This kind of systems offer efficient localization information and considered as cost wise systems, as they require installing an inexpensive microphone to each reference node. Though, each target object requires emitting a voice in order to be sensed by reference nodes. As a result, reference nodes might then be able to detect the position of that target object.

\subsection{Comparing the Existing WSN based Approaches}

In this section, we compare the exiting WSN based approaches which have been discussed in the literature. Table 1 compares the existing localization techniques in terms of accuracy, the requirement of additional hardware (HW), cost, density, deployability, and power consumption.

In terms of tracking "accuracy", GPS and camera-based systems offer reasonable localization accuracy, but GPS tracking systems require attaching an expensive hardware to each target object, which increases cost, complexity, and power consumption for each sensor node.

The "need to additional hardware" is a critical requirement in Threats detection and tracking systems. GPS, and RF-based approaches require each suspect (target) object to be attached with an additional hardware, in order to be localized. While, there is no need for any additional hardware to be attached to the target node when the camera or acoustic-based systems are used.

In terms of "cost", GPS and camera-based systems are considered as high cost localization solutions. GPS systems require attaching a GPS receiver and transceiver to each target object, while an additional camera sensor is required to be added to each reference node in the camera-based tracking systems. In acoustic-based systems, an additional hardware is needed (acoustic sensor), however it's considered as a low cost sensor compared to GPS receiver, transceiver, and camera sensors. RF-based systems offer low cost localization system, as there is no requirement to attach any special device (microphone, ultrasonic, or camera) to reference and target wireless sensor nodes, as this kind of systems depends only on the radio signals transmitted from reference nodes. 
Table 1: A comparison among the localization technologies used in Threats detection

\begin{tabular}{|l|c|c|c|c|}
\hline \multicolumn{1}{|c|}{ Key issue } & GPS & Vision & RF & Acoustic \\
\hline Accuracy & $\begin{array}{c}\text { High localization } \\
\text { accuracy outdoors }\end{array}$ & $\begin{array}{c}\text { High localization } \\
\text { accuracy outdoors } \\
\text { where no obstacles }\end{array}$ & $\begin{array}{c}\text { Low localization } \\
\text { accuracy in both } \\
\text { (indoors \& outdoors) }\end{array}$ & $\begin{array}{c}\text { Reasonable } \\
\text { localization accuracy } \\
\text { in both (indoors \& } \\
\text { outdoors) }\end{array}$ \\
\hline $\begin{array}{l}\text { The requirement of } \\
\text { attaching additional HW } \\
\text { to target node }\end{array}$ & $\begin{array}{c}\text { Requires a receiver } \\
\text { and transceiver } \\
\text { devices }\end{array}$ & $\begin{array}{c}\text { No need to attach any } \\
\text { devices }\end{array}$ & $\begin{array}{c}\text { A simple device is } \\
\text { required }\end{array}$ & $\begin{array}{c}\text { No need to attach any } \\
\text { device }\end{array}$ \\
\hline $\begin{array}{l}\text { The requirement of } \\
\text { attaching additional HW } \\
\text { to reference node }\end{array}$ & No need & $\begin{array}{c}\text { A high resolution } \\
\text { camera is required }\end{array}$ & $\begin{array}{c}\text { A simple device is } \\
\text { required }\end{array}$ & $\begin{array}{c}\text { An acoustic sensor is } \\
\text { required }\end{array}$ \\
\hline Cost & High & High & Low & Moderate \\
\hline $\begin{array}{l}\text { Density of reference } \\
\text { nodes }\end{array}$ & $\begin{array}{c}\text { High density of } \\
\text { reference nodes in } \\
\text { tracking area of } \\
\text { interest }\end{array}$ & Hoderate & Moderate \\
\hline Deployability & $\begin{array}{c}\text { High power } \\
\text { consumption }\end{array}$ & $\begin{array}{c}\text { High power } \\
\text { consumption }\end{array}$ & Low & Medium \\
\hline Power consumption & Flexible outdoors & Moderate \\
\hline
\end{tabular}

GPS tracking systems do not require a high density of sensor nodes to be deployed in the tracking area of interest, as the target's coordinates computed through the values received from the satellites. However, vision-based systems require a high density of reference nodes. The density of reference nodes in the RF-based systems is based on the transmission range. In the acoustic-based systems, the density of reference nodes is based on the voice emitted by the target node.

\subsection{Challenges of WSN Threats Detection and Tracking Systems}

The constrained computation power, battery power, storage capacity, and communication bandwidth of the tiny sensor devices pose challenging problems in the design and deployment of Threats detection and tracking systems. Threats detection and tracking systems pose many challenges when deployed for military applications. One of the key technological challenges is how to track threat objects without attaching any additional device (sensor) to those targets. In RF and GPS-based tracking system, it's essentially to attach a device (GPS receiver and transceiver, or RF module) in order to be able to detect and track Threats' positions.

One more challenge is how to track Threats with the lowest cost possible. Attaching additional hardware (camera, or GPS) to each threat object is inefficient too, because it rises up the tracking system's cost. Using acoustic sensor might reduce the tracking complexity and cost. Acoustic-based systems require attaching a simple acoustic sensor to each reference node, in contrast to GPS and camera-based systems. Now, using an acoustic method to detect and track the position of target objects might overcome some of those challenges.

\section{KEY ISSUES}

Through discussing and analyzing the existing approaches for military tasks, there are many recommendations must to be taken into consideration before designing and implementing a military tracking system. As result, developers need to consider the following issues:

1. Accuracy: the designed system must offer reasonable localization accuracy for Threats detection and tracking systems, as obstacles and walls could be existed in the tracking area of interest, and hence achieves low localization accuracy,

2. Cost: adding a supplementary hardware to hundreds or thousands of nodes is a highly inefficient solution in terms of cost. The localization technique must be cheap in cost in order to be attractive solution for WSN systems

3. Power consumption: in WSN, energy is mainly consumed by three subsystems: signal processing, data transmission, and hardware operations. Consuming less energy in WSN based tracking systems is a primary objective in designing a WSN application, as each sensor node is usually equipped with batteries which could be hard to replace

4. Coverage: the localization system should cover the tracking area of interest in order to sense any suspicious object, and then achieve the goal that was designed for

5. Density of reference node: a high density of reference nodes will increase both the cost and power consumption for WSN system. Therefore, the total number of reference nodes should be as minimum as possible

6. Delay time: the sink node (administrator) must be informed about the threat's position as soon as one of the reference nodes detects its position

7. Deployability: usually, sensor nodes are scattered or deployed using airplanes. The tracking system must be easy to deploy with no need for a hard installation

8. Accessibility: the designed tracking system must be able to work indoors and outdoors. As the system could be deployed where a number of obstacles (such as tree, wall, or vehicle) found in the tracking area of interest

9. Form factor: attaching additional hardware or sensor device to each sensor node will increase the sensor's size. In Threats detection and tracking system, it's critical to keep the sensor node's size as tiny as possible in order to be invisible for Threats objects. 


\section{CONCLUSION AND FUTURE WORK}

Our main objective was to study the exiting localization approaches which can be deployed in WSN and could serve military applications, in order to bring out few key issues for designing and implementing a tracking system to detect and track Threats through border security area. And have outlined how the tracking approach can meet these requirements. It stands for integrating the acoustic device with wireless sensor nodes in order to be able to detect and track the positions of threat objects. The major contribution of this paper includes classification the exiting WSN-based tracking and localization systems.

For future work, we aim to develop and design an acousticbased system for Threats detection and tracking. This kind of systems is based only on emitting a voice by a threat object. Moreover, acoustic sensors are considered as an effective cost solution. Further improvements would be considered to enhance the localization accuracy for the proposed system, by integrating ultrasonic sensor to track the positions of silent Threats.

\section{REFERENCES}

[1] Hamdi, M., Boudriga, N., Obaidat, M. S. 2008. WHOMoVeS: An optimized broadband sensor network for military vehicle tracking, International Journal of Communication Systems, Vol. 21 , Issue 3, pp. 277-300, ISSN:1074-5351.

[2] Son, B.; Her, Y.; Kim, J. 2006. A design and implementation of forest-fires surveillance system based on wireless sensor networks for South Korea Mountains. Int. J. Comput. Sci. Netw. Secur.(IJCSNS) 6, 124-130.

[3] Antoine-Santoni, T., Santucci, J., Gentili, E., De; Silvani, X., Morandini, F. 2009. Performance of a protected wireless sensor network in a fire. Analysis of fire spread and data transmission. Sens. J. 9, 5878-5893

[4] Lloret, J., Garcia, M., Bri, D. and Sendra. S. 2009. A Wireless Sensor Network Deployment for Rural and Forest Fire Detection and Verification. Sensors. Vol. 9 Issue: 11. pp. 8722-8747.

[5] Resch, B., Mittlboeck, M., Girardin, F., Britter, R. and Ratti, C. 2009. Real-time Geo-awareness - Sensor Data Integration for Environmental Monitoring in the City. IN: Proceedings of the IARIA International Conference on Advanced Geographic Information Systems \& Web Services - GEOWS2009, Cancun, Mexico, pp. 92-97.

[6] Alhmiedat, T., and Yang, S 2007. A Survey: Localization and Tracking Mobile Targets through Wireless Sensor Network, PGNet International Conference, ISBN: 1-9025-6016-7.

[7] Shareef, A., Zhu, Y., and Musavi, M. 2008. Localization Using Neural Networks in Wireless Sensor Networks", $1^{\text {st }}$ International Conference on Mobile Wireless MiddleWARE, Operating Systems, and Applications, Innsbruck, Australia.

[8] Takahashi, S., Wong, J., Miyamae, M., Terada, T., Noma, H., Toriyama, T., Kogure, K., and Nishio, S., 2008. A ZigBee based Sensor Node for Tracking People's Locations, ACM International Conference Proceeding Series; Vol. 281, New York, USA.

[9] Alhmiedat, T. A. and Yang, S., 2008. A ZigBee-based Mobile Tracking System Through Wireless Sensor
Networks", Int. Advanced Mechatronic Systems, 1(1), pp. 63-70.

[10] Takahashi, S., Wong, J., Miyamae, M., Terada, T., Noma, H., Toriyama, T., Kogure, K., and Nishio, S. 2008. A ZigBee based Sensor Node for Tracking People's Locations, ACM International Conference Proceeding Series; Vol. 281, New York, USA.

[11] Blumenthal, J., Reichenbach, F. and Timmermann, D. 2005. Position estimation in ad-hoc wireless sensor networks with low complexity, Joint 2nd Workshop on Positioning, Navigation and Communication (WPNC 05) and 1st Ultra-Wideband Expert Talk, pp.41-49.

[12] Blumenthal, J., Grossmann, R., Golatowski, F. and Timmermann, D. 2007. Wighted centroid localization in ZigBee based sensor networks", Folien IEEE International Symposium on Intelligent Signal Processing, WISP, Madrid, Spain.

[13] Blumenthal, J., Reichenbach, F. and Timmermann, D. 2005. Position estimation in ad-hoc wireless sensor networks with low complexity, Joint 2nd Workshop on Positioning, Navigation and Communication (WPNC 05) and 1st Ultra-Wideband Expert Talk, pp.41-49.

[14] He, T., Huang, C., Blum, B. M., Stankovic, J. A. and Abdelzaher, T. F. 2005. Range-Free Localization and its Impact on Large Scale Sensor Networks, ACM Transactions on Embedded Computing System, Vol. 4.

[15] Boukerche, A., Oliveira, H.A.B.F., Nakamura, E.F., and Loureiro, A.A.F. 2007. Localization Systems for Wireless Sensor Networks, IEEE Wireless Communications: Wireless Sensor Networking, pp. 6-12.

[16] Alhmiedat, T. A, and Yang, S., 2011. Tracking Mobile Targets through Wireless Sensor Networks, Lap Lambert Academic Publishing AG \& Co $\mathrm{Kg}$, ISBN 13: 9783844334609.

[17] Padmavathi, G., Shanmugapriya, D., and Kalaivani M. 2010. A Study on Vehicle Detection and Tracking Using Wireless Sensor Networks, Wireless Sensor Network, 173-185.

[18] Psiaki, M.L. 2001. Smoother-Based GPS Signal Tracking in a Software Receiver, Proceedings of ION GPS, pp. 2900-2913.

[19] Chadil, N., Russameesawang, A., and Keeratiwintakorn, P. 2008. Real-Time Tracking Management System using GPS, GPRS and Google Earth, in Proc of the 5th International Conference on Electrical Engineering, Telecommunications and Information Technology, Thailand.

[20] Handcock, R., Swain, D., Bishop-Hurley, G., Patison, K., Wark, T., Valencia, P., Corke, P., O'Neill, C. 2009. Monitoring Animal Behaviour and Environmental Interactions Using Wireless Sensor Networks, GPS Collars and Satellite Remote Sensing. Sensors 9, 35863603.

[21] Ding, F., Song, G., Yin, K., Li, J., and Song. A. 2009. A GPS-enabled wireless sensor network for monitoring radioactive materials", Sensors and Actuators A: Physical 155, 210-215.

[22] Matamoros, J. M., Martinez, J. R., and Ollero, A. 2009. Cooperative localization and tracking with a camera- 
based WSN. Proceedings of the 2009 IEEE International Conference on Mechatronics, Malaga, Spain, April.

[23] Chen, W. T., Chen, P.Y., Lee, W.S., and Huang, C.F. 2008. Design and implementation of a real time video surveillance system with wireless sensor networks, in Vehicular Technology Conference. VTC Spring IEEE, p. 218

[24] Paniga, S., Borsani, L., Redondi, A., Tagliasacchi, M., and Cesana, M. 2010. Experimental Evaluation of a Video Streaming System for Wireless Multimedia Sensor Networks, The $10^{\text {th }}$ IFIP Annual Mediterranean Ad Hoc Networking Workshop, Milano, Italy.

[25] Jung, B. and Sukhatme, G.S. 2001. Tracking Multiple Moving Targets using a Camera and Laser Rangefinder, Technical Report IRIS-01-397, Institute for Robotics and Intelligent Systems (IRIS), University of Southern California.

[26] Reichenbach, F., Blumenthal, J. and Timmermann, D. 2006. Improved precision of coarse grained localization in wireless sensor networks, 9th DSD Conference, Dubrovnik, Croatia, pp.630-637.

[27] Reichenbach, F. and Timmermann, D. 2006. Indoor Localization with low complexity in wireless sensor networks, Proceeding of the IEEE (INDIN'06), pp.10181023 .

[28] Paschos, G. S., Vagenas, E. D., and Kotsopoulos, S. A. 2005. Real-time localization for wireless sensor networks with multiple reference transmissions, in Proceedings of the 5th International Network Conference (INC '05), Island of Samos, Greece.

[29] Li, B., Salter, J., Dempster, A. G., and Rizos, C. 2006. Indoor Positioning Techniques Based on Wireless LAN", Proceedings of AusWireless, Sydney, Australia.

[30] Li, B., Dempster, A., Rizos, C., and Barnes, J. 2005. Hybrid Method for Localization using WLAN, Spatial Sciences Conference, Melbourne, Australia.
[31] Small, J., Smaliagic, A., and Siewiorek, D. 2000. Determining user Locations For Context Aware Computing Through the Use of a Wireless LAN Infrastructure, Institute for Complex Engineered Systems, Carnegie Mellon University, Pittsburgh, PA 15213, USA.

[32] Ahmad, U., Gavrilov, A., Nasir, U., Iqbal, M., Seong, J. C., and Sungyoung, L. 2006. In-building Localization using Neural Networks, IEEE International Conference of Intelligent Systems, Islamabad, Pakistan.

[33] Battiti, R., Nhat, T. L., and Villani, A. 2002. Locationaware computing: a neural network model for determining location in wireless LANs, Tech. Rep. DIT02-0083, University of Trento, Trento, Italy.

[34] Simon, G., and Sujbert, L. 2006. Acoustic source localization in sensor networks with low communication bandwidth, International Workshop on Intelligent Solutions in Embedded Systems.

[35] Werner-Allen, G., Johnson, J., Ruiz, M., Lees, J., and Welsh. M. 2005. Monitoring volcanic eruptions with a wireless sensor network, In Proc. Second European Workshop on Wireless Sensor Networks (EWSN'05).

[36] Ko, J. H., Shin, J., Kwon, S. and Kim, C. 2008 Localization of sensor nodes in underwater acoustic sensor networks using two reference points. International Conference on Information Networking, ICOIN.

[37] Kushwaha, M., Amundson, I., Volgyesi, P., Ahammad, P., Simon, G., Koutsoukos, X., Ledeczi, A., Sastry, S 2008. Multi-modal target tracking using heterogeneous sensor networks. In: Proc. of ICCCN.

[38] Meesookho, C., and Mitra, U. 2008. On energy-based acoustic source localization for sensor networks, IEEE Trans. Signal Process., vol. 56, no. 1, pp. 365-377.

[39] Collier, T.C., Kirschel, A.N.G. and Taylor, C.E. 2010 Acoustic localization of antbirds in a Mexican rainforest using a wireless sensor network. J. Acoust. Soc. Am. 128: $182-189$. 